\title{
Semi-central In+In collisions and Brown-Rho scaling
}

\author{
V.V. Skokov and V.D. Tonee田 \\ Bogoliubov Laboratory of Theoretical Physics, \\ Joint Institute for Nuclear Research, \\ 141980, Dubna, Russia
}

\begin{abstract}
In connection with the claim made at the Quark Matter 2005 Conference that the Brown-Rho scaling is ruled out by NA60 data we consider dimuon production from semi-central In+In collisions in a full dynamical model. It is shown that if only a modification of the density-dependent $\rho$-mass is allowed, the maximum of dimuon invariant mass spectra is only slightly below experimental one. The additional inclusion of the temperature-dependent modification factor shifts the spectrum maximum toward lower invariant masses making calculation results incompatible with data. A further study is needed to disentangle the BR dropping $\rho$ mass scaling and strong broadening.
\end{abstract}

Because of weak interaction, dileptons play an exceptional role among different probes providing information on a state of highly compressed and hot nuclear matter formed in relativistic heavy ion collisions. Generally, a dilepton yield depends on both global properties of matter constituents (hadrons and/or quarks, gluons) defined by the equation of state and individual constituent properties related to their in-medium modification. The analysis of the $e^{+} e^{-}$invariant mass spectra from central $\mathrm{Pb}+\mathrm{Au}$ collisions at the bombarding energy $E_{l a b}=158 \mathrm{~A}$ $\mathrm{GeV}$, measured by the CERES Collaboration, for certain shows an excess radiation in the range of invariant dilepton masses $0.2 \lesssim M \lesssim 0.7 \mathrm{GeV}$. A possible interpretation of this excess was given in terms of a strong inmedium $\rho$-meson modification (see review articles 1, 2]). Various scenarios of hadron modification were proposed. However, low statistics, insufficient mass resolution, and large signal/background ratio in the CERES experiments did not allow one to discriminate these scenarios, in particular those based on the Brown-Rho (BR) scaling hypothesis [3] assuming a dropping $\rho$ mass and on a strong broadening as found in the many-body approach by Rapp and Wambach [2, 4].

The proposed NA60 experiment with the muon detector, zero degree calorimeter and the refined target area does not seem to suffer from these CERES shortcomings. The first NA60 results for $\mu^{+} \mu^{-}$pair production in In+In collisions have recently been presented at the Quark Matter 2005 Conference [5] (see also Fig.1 in Ref. [6])). From comparison of these results with Rapp's theoretical predictions it was declared that the "BR scaling is ruled out by NA60" dimuon data whereas the many-body approach gives a reasonable agreement. This claim immediately raised an objection by Brown and Rho. In their two letters [6, 7] they noted three points that were neglected or simplified in the calculation of the dropping mass scenario which make it having nothing to do with that they believe BR scaling $[\underline{3}, \underline{8}$ to be.

All three key points stated in $[\underline{6}, 7]$ may be essential in

*Electronic address: vvskokov@theor.jinr.ru

${ }^{\dagger}$ Electronic address: toneev@theor.jinr.ru the description of dilepton production but still have not been properly taken into account in the works available now in the literature. This also concerns this letter : We assume the validity of the vector dominance and do not discuss "sobar" excitations. We basically concentrate on the influence of the temperature and baryon density dependence in the form of scaling. Moreover, nuclear interaction dynamics is presented in detail to make clear which states mainly contribute to the observed dimuon yield as well as the model parameters used.

The dynamics of heavy ion collisions is treated in terms of a hybrid model where the initial interaction stage is described by the transport Quark Gluon String Model (QGSM) 9] and the subsequent stage is considered as an isoentropic expansion. The latter stage is calculated within the relativistic 3D hydrodynamics [10] allowing different equations of state. In our work, the mixed phase Equation of State (EoS) [1] is applied. This thermodynamically consistent EoS uses the modified Zimanyi mean-field interaction for hadrons and also includes interaction between hadron and quark-gluon phases, which results in a cross-over deconfinement phase transition. In addition to Ref. 11], the hard thermal loop term was self-consistently added to the interaction of quarks and gluons to get the correct asymptotics at $T>T_{c}$ and reasonable agreement of the model results with lattice QCD calculations at finite temperature $T$ and chemical potential $\mu_{B}$ [12].

The ratio between entropy $S$ and total participant baryon charge $Q_{B}$, is shown in Fig.1 for In+In collisions at the impact parameter $b=4 \mathrm{fm}$ and bombarding energy $158 A \mathrm{GeV}$ estimated within QGSM. Being calculated on a large $3 \mathrm{D}$ grid, this ratio is less sensitive to particle fluctuation as compared to entropy itself. Small values of $Q_{B}$ at the very beginning of interaction result in large values of the $S / Q_{B}$ ratio. It is clearly seen that for $t_{\text {kin }} \gtrsim 1.3 \mathrm{fm} / c$ this ratio is practically kept constant and this stage may be considered as isoentropic expansion.

To proceed from kinetics to hydrodynamics, we evaluate conserving components of the energy-momentum tensor $T_{00}, T_{01}, T_{02}, T_{03}$ and baryon density (the zero component of the baryon current) within QGSM at the moment $t_{k i n}=1.3 \mathrm{fm} / c$ in every cell on the $3 \mathrm{D}$ grid. This state is 


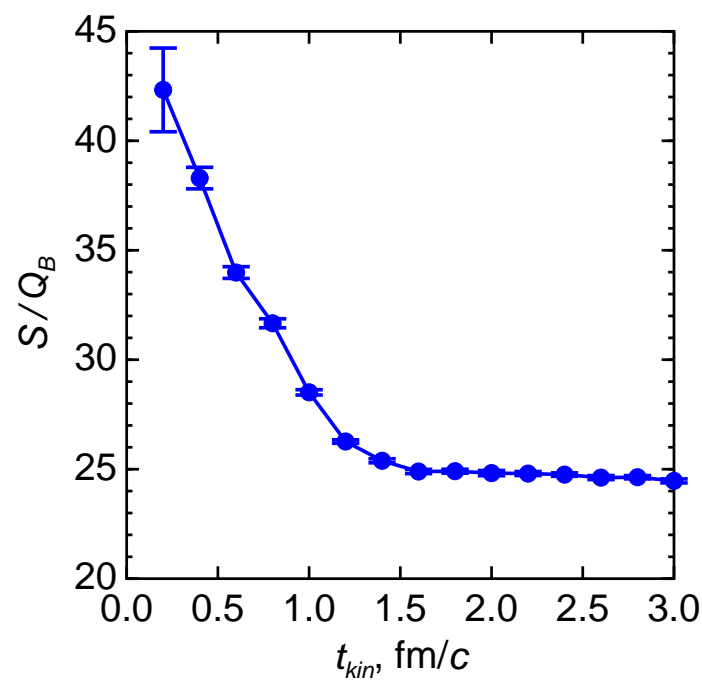

FIG. 1: (Color online) Temporal dependence of entropy $S$ per baryon charge $Q_{B}$ of participants for semi-central In+In collision at $E_{l a b}=158 \mathrm{~A} \mathrm{GeV}$.

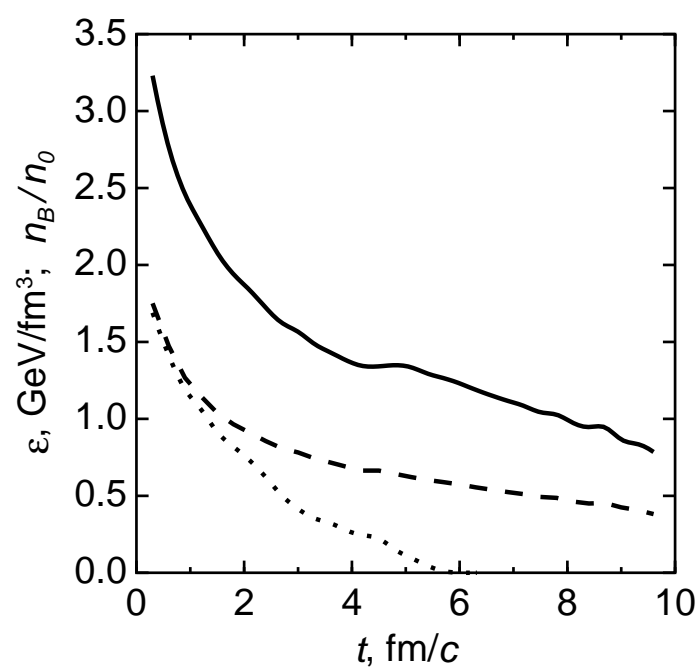

FIG. 2: The average energy (solid line) and baryon (dashed) densities of an expanding fireball formed in In+In collisions. Dotted line shows a contribution of quarks and gluons to the energy density.

treated as an initial state for subsequent hydrodynamic evolution of a fireball. The time dependence of average thermodynamic quantities is presented in Figs.2,3.

As is seen, the average (over the whole excited system) initial energy density and the compression ratio, $n_{B} / n_{0}$, are not so high, about $3.2 \mathrm{GeV} / \mathrm{fm}^{3}$ and 1.7 , respectively. These values are by a factor of $\sim 2$ lower than those reached in central $\mathrm{Pb}+\mathrm{Au}$ collisions at the same energy in the CERES experiments. Both quantities fall down in time and exhibit a weak structure related to the softest point of the mixed phase EoS [11]. The depicted contribution of quark-qluon degrees of freedom to energy density is not large and the muon creation from this phase will be neglected below. The temperature evolution (Fig.3) looks quite similarly. The calculated critical temperature at the considered finite $\mu_{B}$ is about $160 \mathrm{MeV}$, so the evolution starts slightly above this value. The end point of the temperature evolution curve is about $135 \mathrm{MeV}$ and is governed by freeze-out. In our model, the freeze-out occurs locally and continuously during the whole evolution. The freeze-out condition is that the local energy density (including 6 neighbor cells) is below a certain value $\varepsilon_{f r}$ which was fixed by describing pion multiplicity in central $\mathrm{Pb}+\mathrm{Pb}$ collisions at the maximal SPS energy [10]. One should note that the 1D Lorentz invariant Bjorken hydrodynamics 13] predicts an essentially faster fall down and, therefore, a much shorter hydrodynamic evolution time.

To find observable dilepton characteristics, one should integrate the emission rate over the whole time-space $x \equiv(t, \mathbf{x})$ evolution, add the contribution from the freezeout surface (hadron cocktail), and take into account the experimental acceptance. To simplify our task, we consider only the main channel $\pi \pi \rightarrow l^{+} l^{-}$. In this case the dilepton emission rate is

$$
\begin{aligned}
\frac{d^{4} N}{d^{4} q} & =-\int d^{4} x \mathcal{L}(M) \frac{\alpha^{2}}{\pi^{3} q^{2}} f_{B}\left(q_{0}, T(x)\right) \\
& \times \operatorname{Im} \Pi_{e m}\left(q, T(x), \mu_{b}(x)\right),
\end{aligned}
$$

where the integration is carried out over the whole grid and time from $t=0$ till the local freeze-out moment. Here $q^{2}=M^{2}=q_{0}^{2}-\mathbf{q}^{2}$, the Bose distribution function is defined as

$$
f_{B}\left(q_{0}, T(x)\right)=\left(e^{q_{0} / T(x)}-1\right)^{-1}
$$

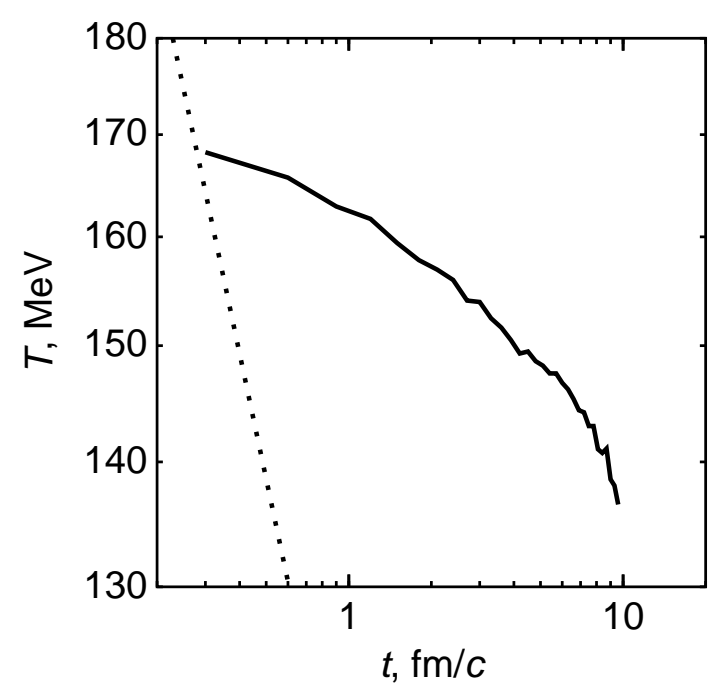

FIG. 3: Evolution of the average temperature. The dotted line corresponds to the Bjorken regime with ultrarelativistic ideal gas EoS. 
and lepton kinematic factor is

$$
\mathcal{L}(M)=\left(1+2 \frac{m_{l}^{2}}{M^{2}}\right) \sqrt{1-4 \frac{m_{l}^{2}}{M^{2}}}
$$

with the lepton mass $m_{l}$. If the pole shift is neglected $\left(m_{\rho}^{\text {pole }}=m_{\rho}\right)$, the imaginary part of the electromagnetic current correlation function, integrated over $\mathbf{q}$, for free $\pi \pi$ annihilation reads

$$
\operatorname{Im} \Pi_{e m}(M)=\frac{m_{\rho}^{4}}{g^{2}} \frac{\operatorname{Im} \Pi}{\left(M^{2}-m_{\rho}^{2}\right)^{2}+(\operatorname{Im} \Pi)^{2}} .
$$

The imaginary part of the $\rho$-meson self-energy in the oneloop approximation is

$$
\operatorname{Im} \Pi=-\frac{g_{\rho \pi \pi}^{2}}{48 \pi} \frac{\left(M^{2}-4 m_{\pi}^{2}\right)^{3 / 2}}{M},
$$

where the parameters are defined in vacuum [14]:

$$
g_{\rho \pi \pi}=6.05 ; g=5.03, m_{\rho}=770 \mathrm{MeV} .
$$

To simulate the Brown-Rho scaling, we modify the inmedium masses in eq. (4), (15) estimated according to the QCD sum rules by Hatsuda and Lee [15, 16]

$$
m_{\rho} \rightarrow m_{\rho}^{*}(x)=m_{\rho}\left(1-0.15 \cdot n_{B}(x) / n_{0}\right)
$$

and simultaneously apply the same modification to the vector dominance coupling $m_{\rho}^{2} / g \rightarrow m_{\rho}^{* 2} / g^{*}$ [17], as it was suggested in [6, 7].

Rapp's results cited in ref. [5] were obtained using a different assumption on the in-medium mass [2]

$$
m_{\rho}^{*}(x)=m_{\rho}\left(1-0.15 \cdot \frac{n_{B}(x)}{n_{0}}\right)\left(1-\left[\frac{T(x)}{T_{c}}\right]^{2}\right)^{0.3}
$$

The temperature dependence in eq. (8) is motivated by the T-dependence of quark condensate.

Dimuon invariant mass spectra from $\mathrm{In}+\mathrm{In}$ collisions are presented in Fig.4 for the $\pi \pi$ channel. Only the muon rapidity cut $3<y_{l a b}<4$ is taken into account in our calculations. The NA60 experimental points demonstrate quite a high resolution in dimuon mass; however, their absolute normalization is lost. As is seen, if the $T$-dependent dropping mass (8) is assumed, our curve is strongly shifted toward the low-mass region which is in qualitative agreement with the Rapp result [5]. In the case of the density dependent $\rho$ mass scaling (7) the maximum position is only slightly below experimental ones and this phenomenological scenario cannot unambiguously be "ruled out". To compare properly dileptons from the pion annihilation with experiment, the contribution of free $\rho$-meson decay should be added. A number of these $\rho$-mesons was estimated as thermodynamical emission from frozen-out cells, according to the general procedure described in [18]. In the last case the agreement with experiment will be even better if the muon contribution from the free $\rho$ decay is taken into account. Note

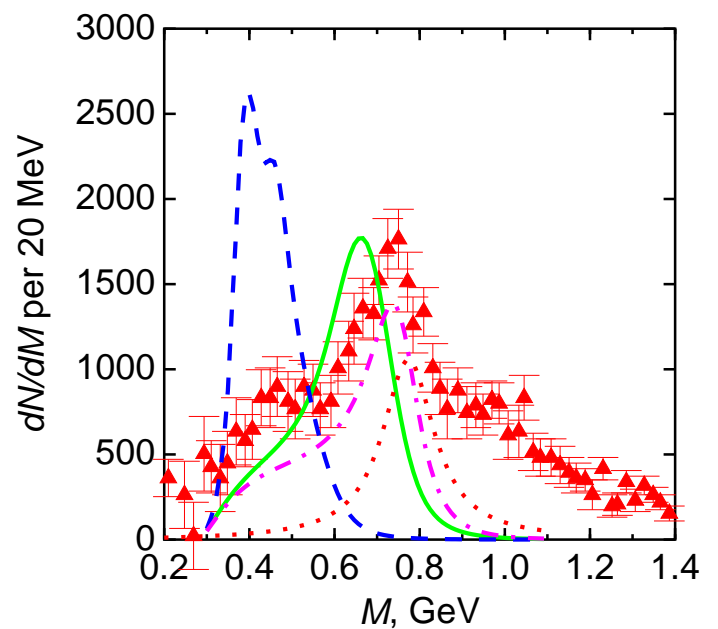

FIG. 4: (Color online) Invariant mass distribution of dimuons from semi-central In+In collisions at the beam energy 158 $A \mathrm{GeV}$. Experimental points are from 5]. Solid and dashed curves are calculated using the $\rho$-mass modification factors (7) and (8), respectively. The dash-dotted curve neglects any in-medium modification. The dotted line indicates the hydrodynamically calculated $\rho$-meson decay at the freeze-out.

that the calculated solid and dotted lines in Fig.4 are multiplied by the same normalization factor and, therefore, the shown ratio between the signal and free $\rho$-meson decay is kept. Muon emission from pion annihilation in the vacuum case very nearly follows the measured points; however, as noted above, the observable quantity is not this spectrum but its sum with the hadron decay cocktail (dotted line in Fig.4). It is of interest that making use of the not modified $m_{\rho}^{2} / g$ ratio increases the muon yield by a factor of $\sim 2$, as expected by [6, 7], but it is not visible in normalized data because the spectrum shape is weakly changed.

Finally, the in-medium modification of the $\rho$-meson mass based on the $T$-dependent scaling (8) is hardly compatible with the NA60 data. As noted in [6, 7], a more realistic case corresponds to neglecting any temperature dependence. This is indeed so, as follows from our results. To disentangle BR dropping $\rho$ mass scaling [3] and strong broadening 2, 4], further detailed theoretical investigations (e.g. see [19]) and a comparison with measured absolute muon yield taking properly into account the experimental acceptance are needed.

We are grateful to Yu. B. Ivanov and V. N. Russkih for numerous discussions of details of hydrodynamical code, freeze-out procedure, and account for experimental acceptance. We are thankful to R. Rapp for critical remarks and S. Damjanovic for discussion of experimental data. One of us (VS) thanks organizers of the QM2005 conference for making his participation possible. This work was supported in part by DFG (project 436 RUS 113/558/0-3) and RFBR (grant 06-02-04001). 
[1] W. Cassing and E.L. Bratkovskaya, Phys. Rep. 308, 65 (1999).

[2] R. Rapp and J. Wambach, Adv. Nucl. Phys. 25, 1 (2000).

[3] G.E. Brown and M. Rho, Phys. Rev. Lett. 66, 2720 (1991).

[4] R. Rapp, G. Chanfray and J. Wambach, Phys. Rev. Lett. 76, 368 (1996) hep-ph/9508353.

[5] S. Damjanovic et al., nucl-ex/0510044

[6] G.E. Brown and M. Rho, nucl-th/0509001

[7] G.E. Brown and M. Rho, nucl-th/0509002

[8] G.E. Brown and M. Rho, Phys. Rept. 396, 1 (2004).

[9] N.S. Amelin, K.K. Gudima, S.Y. Sivoklokov and V.D. Toneev, Sov. J. Nucl. Phys. 52, 172 (1990) [Yad. Fiz. 52, 272 (1990)]; N.S. Amelin, E.F. Staubo, L.P. Csernai, V.D. Toneev and K.K. Gudima, Phys. Rev. C 44, 1541 (1991); V.D. Toneev, N.S. Amelin, K.K. Gudima and S.Yu. Sivoklokov, Nucl.Phys. A519, 463c (1990).

[10] V.V. Skokov and V.D. Toneev, JINR-P2-2005-95 (2005).
[11] V.D. Toneev, E.G. Nikonov, B. Friman, W. Nörenberg, and K. Redlich, Eur. Phys. J. C32, 399 (2004) hep-ph/0308088.

[12] Z. Fodor, Nucl. Phys. A715, 319 (2003); F. Csikor, G.I. Egri, Z. Fodor, S.D. Katz, K.K. Szabo, and A.I. Toth, JHEP 405, 46 (2004).

[13] J.P. Bjorken, Phys. Rev. D 27, 140 (1984).

[14] F. Klingl, N. Kaiser and W. Weise, Z. Phys. A 356, 193 (1996) hep-ph/9607431.

[15] T. Hatsuda and S.H. Lee, Phys. Rev. C 46, R34 (1992).

[16] To be precise, we note that Hatsuda and Lee result was $m_{\rho}^{*}=m_{\rho}\left(1-0.18 \cdot n_{B} / n_{0}\right)$. In our work the factor 0.15 is used instead of 0.18 to be consistent with [5].

[17] Note that this substituton really means some suppression of the vector dominance $[6,7,[8]$.

[18] Yu.B. Ivanov, V.N. Russkikh and V.D. Toneev, nucl-th/0503088

[19] J. Ruppert, T. Renk and B. Müller, hep-ph/0509134 